\title{
A corrected quadrature formula and applications
}

\author{
Nenad Ujević* $\quad$ A. J. Roberts ${ }^{\dagger}$ \\ (Received 26 March 2003; revised 13 February 2004)
}

\begin{abstract}
A straightforward three-point quadrature formula of closed type is derived that improves on Simpson's rule. Just using the additional information of the integrand's derivative at the two endpoints we show the error is sixth order in grid spacing. Various error bounds for the quadrature formula are obtained to quantify more precisely the errors. Applications in numerical integration are given. With these error bounds, which are generally better than the usual Peano bounds, the composite formulas can be applied to integrands with lower order derivatives.
\end{abstract}

*Department of Mathematics, University of Split, Teslina 12/III, 21000 Split, Croatia. mailto: ujevic@mapmf .pmfst.hr

$\dagger^{\dagger}$ Dept Maths \& Computing, University of Southern Queensland, Toowoomba, Queensland 4352, Australia. mailto:aroberts@usq.edu.au

See http://anziamj.austms.org.au/V45/E051/home.html for this article, (C) Austral. Mathematical Soc. 2004. Published February 17, 2004 ISSN 1446-8735 


\section{Contents}

1 Introduction

E42

2 Finite differences derive the modified Simpson's rule

E44

3 Further error analysis

E46

4 Applications in numerical integration

E51

References

E54

\section{Introduction}

In recent years some authors have considered so called perturbed (corrected) quadrature rules. For examples: Cerone and Dragomir [1,2] considered the corrected midpoint and trapezoid quadrature rules; and with Agarwal [3, 4] they considered Simpson's rule.

As a specific introductory example, consider a correction to the midpoint rule. The classical midpoint rule has the form

$$
\int_{a}^{b} f(x) d x=(b-a) f\left(\frac{a+b}{2}\right)+R(f),
$$

where $R(f)$ is the remainder term (error) of this rule. A perturbed (corrected) rule involves the derivative at the endpoints:

$$
\int_{a}^{b} f(x) d x=(b-a) f\left(\frac{a+b}{2}\right)+\frac{(b-a)^{2}}{24}\left[f^{\prime}(b)-f^{\prime}(a)\right]+R_{1}(f),
$$

where $R_{1}(f)$ is the remainder term (error) of this rule. The following properties have analogues in the work we report on Simpson's rule: 
1. the original rule (1) is exact for polynomials of degree 1 , while the perturbed rule is exact for polynomials of degree 3 ,

2. estimates of the errors are respectively

$$
\begin{aligned}
|R(f)| & \leq \frac{(b-a)^{3}}{24} M_{2}, \\
\left|R_{1}(f)\right| & \leq \frac{(b-a)^{3}}{18 \sqrt{3}} M_{2},
\end{aligned}
$$

where $\left|f^{\prime \prime}(t)\right| \leq M_{2}, t \in[a, b]$ - the bound (4) is better than (3);

3. a corresponding composite quadrature formula, for the corrected rule, has only one additional term, with respect to a composite formula for the original rule,

4. the corrected composite formula has a better estimation of error than the original composite formula (a consequence of 2).

Indeed another bound is

$$
\left|R_{1}(f)\right| \leq \frac{7 M_{4}}{5760}(b-a)^{5},
$$

where $\left|f^{(4)}(t)\right| \leq M_{4}$ for $t \in[a, b]$.

The above properties are valid for similar corrected rules (for example, for the corrected trapezoid rule) [2]. However, we cannot correct all quadrature rules such that all the properties 1-4 hold. In Section 2 we show that the well-known Simpson's rule does have a simple endpoint correction, but that the quadrature weights have to be modified as well, see (8). We highlight some advantages of the corrected rule over the Simpson's rule. In Section 3 various error bounds of this rule are obtained. These error bounds are generally, but not always, better than the usual Peano error bounds. In Section 4 applications in numerical integration are given. An illustrative example demonstrates that the modified rule gives better results than Simpson's rule. 


\section{Finite differences derive the modified Simpson's rule}

We modify Simpson's rule for integration. First, we consider integration rules formed over just two consecutive subintervals, each of length $h$, and derive endpoint modifications. This is analogous to the improvement to (1) achieved by the inclusion of endpoint derivative information in (2). Second, this modified Simpson's rule is straightforwardly summed to apply to an integration over many subintervals. In later sections we rederive these formula with less restrictions on the integrand and with error bounds rather than just leading order estimates.

Theorem 1 For $C^{\infty}[a, b]$ integrands $f$,

$$
\begin{aligned}
\int_{a}^{b} f(x) d x= & \frac{b-a}{30}\left[7 f(a)+16 f\left(\frac{a+b}{2}\right)+7 f(b)\right] \\
& -\frac{(b-a)^{2}}{60}\left[f^{\prime}(b)-f^{\prime}(a)\right]+\bar{R}(f),
\end{aligned}
$$

where the error term is, to a leading order estimate,

$$
\bar{R}(f) \approx \frac{(b-a)^{6}}{302400}\left[f^{v}(b)-f^{v}(a)\right] .
$$

Example: As a simple illustrative example, consider $\int_{-1}^{1} e^{x} d x=e-1 / e=$ 2.3504. Simpson's rule estimates the integral as approximately $(e+4+$ $1 / e) / 3=2.3621$, in error by about 0.01 . However, the modified Simpson's rule (5) estimates the integral as $(6 e+16+8 / e) / 15=2.3502$, which has an error about two orders of magnitude smaller. This modification to Simpson's rule can be very effective. 
Proof: Consider integrating $f$ over two consecutive intervals in a regular grid of points $x_{j}$ with grid spacing $h$. Identify $a=x_{j-1}, b=x_{j+1}$ and hence $x_{j}=(a+b) / 2$ is the midpoint. Following [5, p65], we write the analysis in terms of centred difference and mean operators, $\delta f_{j}=f_{j+1 / 2}+f_{j-1 / 2}$ and $\mu f_{j}=\left(f_{j+1 / 2}-f_{j-1 / 2}\right) / 2$ respectively, and the differentiation operator denoted by $\partial$. Then the integral [5, p69]

$$
\frac{1}{2 h} \int_{x_{j-1}}^{x_{j+1}} f(x) d x=(h \partial)^{-1} \mu \delta f_{j} .
$$

To derive a three point integration rule with endpoint corrections such as (5), the above right-hand side must be in the form $\left[1+\alpha \delta^{2}+\mu \delta \beta(h \partial)\right] f_{j}$ for some constant $\alpha$ and some function $\beta(h \partial)$ : $\left[1+\alpha \delta^{2}\right] f_{j}$ symmetrically involves $f_{j}$ and $f_{j \pm 1}$ alone; and $\mu \delta \beta(h \partial) f_{j}$ only involves the derivatives of $f$ at the endpoints $x_{j \pm 1}$. Thus we rearrange the operator equation

$$
\begin{aligned}
& (h \partial)^{-1} \mu \delta=1+\alpha \delta^{2}+\mu \delta \beta(h \partial) \\
\Leftrightarrow & \beta(h \partial)=\frac{1}{h \partial}-\frac{1}{\mu \delta}-\alpha \frac{\delta}{\mu} \\
\Leftrightarrow & \beta(h \partial)=\frac{1}{h \partial}-\frac{1}{\sinh h \partial}-\alpha 2 \tanh \frac{1}{2} h \partial,
\end{aligned}
$$

as $\delta=2 \sinh \frac{1}{2} h \partial$ and $\mu=\cosh \frac{1}{2} h \partial$ [5, p65]. Choosing this particular function $\beta(h \partial)$ would generate a rule in the interior together with end point corrections that would give an exact quadrature formula (for all $\alpha$ ). However, the infinite derivatives required are not practical.

We chose $\alpha$ to generate an accurate rule that only needs to know function values and the end point derivatives. The approach is to expand this function $\beta(h \partial)$ in powers of small $h \partial$ to see

$$
\beta=\left(-\alpha+\frac{1}{6}\right) h \partial+\left(\frac{1}{12} \alpha-\frac{7}{360}\right)(h \partial)^{3}+\left(-\frac{1}{120} \alpha+\frac{31}{15120}\right)(h \partial)^{5}+\cdots .
$$

Observe as an aside that choosing $\alpha=1 / 6$ eliminates the first derivative term in $\beta$ leading to the familiar Simpson's rule with an error determined by 
the neglected parts of $\beta$, namely the end-point contributions $-\mu \delta(h \partial)^{3} / 180+$ $\ldots .{ }^{1}$ Instead we choose $\alpha=7 / 30$ to eliminate the third derivative term in $\beta$ :

$$
\begin{aligned}
\int_{x_{j-1}}^{x_{j+1}} f(x) d x= & 2 h(h \partial)^{-1} \mu \delta f_{j} \\
= & 2 h\left[1+\frac{7}{30} \delta^{2}\right] f_{j}+2 h \mu \delta\left[-\frac{1}{15} h \partial+\frac{1}{9450} h \partial^{5}+\cdots\right] f_{j} \\
= & \frac{h}{15}\left[7 f_{j-1}+16 f_{j}+7 f_{j+1}\right] \\
& -\frac{h^{2}}{15}\left[f_{j+1}^{\prime}-f_{j-1}^{\prime}\right]+\frac{h^{6}}{4725}\left[f_{j+1}^{v}-f_{j-1}^{v}\right]+\cdots .
\end{aligned}
$$

Substitute $h=(b-a) / 2$ to reproduce (5) and its leading order error (6).

Corollary 2 Apply (7) to $n$ consecutive pairs of intervals from say $a=x_{0}$ to $b=x_{2 n}$ and sum to immediately deduce that for $C^{\infty}[a, b]$ integrands $f$

$$
\int_{a}^{b} f(x) d x=\frac{h}{15} \sum_{j=1, j \text { odd }}^{2 n-1}\left[7 f_{j-1}+16 f_{j}+7 f_{j+1}\right]-\frac{h^{2}}{15}\left[f^{\prime}(b)-f^{\prime}(a)\right]+\bar{R}(f) .
$$

where the error is to leading order

$$
\bar{R}(f) \approx \frac{h^{6}}{4725}\left[f^{v}(b)-f^{v}(a)\right]
$$

See that these simple modifications to Simpson's rule generate an integration method with error $\mathcal{O}\left(h^{6}\right)$.

\section{$3 \quad$ Further error analysis}

For the sake of simplicity, we first consider the error analysis for the quadrature formula (5) on the interval $[0,1]$. Then we easily transform obtained

${ }^{1}$ Thus Simpson's rule has no simple end correction involving just the first derivative of the integrand, of the type described in (2) for the mid-point rule, instead the end correction would necessarily involve the third derivative. 
results to an arbitrary interval. The formula $(5)$ on the interval $[0,1]$ has the form

$$
\int_{0}^{1} f(x) d x=\frac{7 f(0)+16 f\left(\frac{1}{2}\right)+7 f(1)}{30}-\frac{f^{\prime}(1)-f^{\prime}(0)}{60}+R_{k}(f),
$$

for $k=2,3,4,5,6$. Using the Peano Kernel Theorem we find the following Peano kernels

$$
T_{k}(x)= \begin{cases}(-1)^{k} P_{k}(x), & x \in\left[0, \frac{1}{2}\right) ; \\ (-1)^{k} Q_{k}(x), & x \in\left[\frac{1}{2}, 1\right] ;\end{cases}
$$

for $k=2,3,4,5,6$, where

$$
\begin{array}{ll}
P_{2}(x)=\frac{1}{2} x^{2}-\frac{7}{30} x+\frac{1}{60}, & Q_{2}(x)=\frac{1}{2} x^{2}-\frac{23}{30} x+\frac{17}{60}, \\
P_{3}(x)=\frac{1}{3 !} x\left(x-\frac{1}{5}\right)\left(x-\frac{1}{2}\right), & Q_{3}(x)=\frac{1}{3 !}(x-1)\left(x-\frac{1}{2}\right)\left(x-\frac{4}{25}\right), \\
P_{4}(x)=\frac{1}{4 !} x^{2}\left(x-\frac{1}{3}\right)\left(x-\frac{3}{5}\right), & Q_{4}(x)=\frac{1}{4 !}(x-1)^{2}\left(x-\frac{2}{3}\right)\left(x-\frac{2}{5}\right), \\
P_{5}(x)=\frac{1}{5 !} x^{3}\left(x-\frac{1}{2}\right)\left(x-\frac{2}{3}\right), & Q_{5}(x)=\frac{1}{5 !}(x-1)^{3}\left(x-\frac{1}{2}\right)\left(x-\frac{1}{3}\right), \\
P_{6}(x)=\frac{1}{6 !} x^{4}\left(x^{2}-\frac{7}{5} x+\frac{1}{2}\right), & Q_{6}(x)=\frac{1}{6 !}(x-1)^{4}\left(x^{2}-\frac{3}{5} x+\frac{1}{10}\right) .
\end{array}
$$

We have

$$
R_{k}(f)=\int_{0}^{1} T_{k}(x) f^{(k)}(x) d x,
$$

for $k=2,3,4,5,6$. We also have

$$
\begin{gathered}
\int_{0}^{1} T_{k}(x) d x=0,, \quad k=2, \ldots, 5, \\
\int_{0}^{1}\left|T_{k}(x)\right| d x=C_{k}, \quad k=2, \ldots, 6,
\end{gathered}
$$

where

$$
\begin{aligned}
C_{2} & =\frac{19 \sqrt{19}}{10125}, \quad C_{3}=\frac{253}{360000}, \quad C_{4}=\frac{1}{14580}, \quad C_{5}=\frac{1}{115200}, \\
C_{6} & =\frac{1}{604800},
\end{aligned}
$$


and

$$
\max _{x \in[0,1]}\left|T_{k}(x)\right|=B_{k}, \quad k=2,3,4,5,
$$

where

$$
B_{2}=\frac{1}{40}, \quad B_{3}=\frac{7}{20250}+\frac{19 \sqrt{19}}{81000}, \quad B_{4}=\frac{1}{5760}, \quad B_{5}=\frac{1}{58320} .
$$

Theorem 3 Let $f \in C^{k}(0,1)$ and let $\gamma_{k}, \Gamma_{k}$ be real numbers such that $\gamma_{k} \leq$ $f^{(k)}(x) \leq \Gamma_{k}, x \in[0,1], k=2,3,4,5$. Let $S_{k}=f^{(k)}(1)-f^{(k)}(0), k=$ $1,2,3,4$. Then we have

$$
\begin{aligned}
& \left|R_{k}(f)\right| \leq \frac{\Gamma_{k}-\gamma_{k}}{2} C_{k}, \quad k=2, \ldots, 5, \\
& \left|R_{k}(f)\right| \leq\left(S_{k-1}-\gamma_{k}\right) B_{k}, \quad k=2, \ldots, 5, \\
& \left|R_{k}(f)\right| \leq\left(\Gamma_{k}-S_{k-1}\right) B_{k}, \quad k=2, \ldots, 5,
\end{aligned}
$$

where $R_{k}(f)$ are defined by (12), $C_{k}$ are defined by (15) and $B_{k}$ are defined by (18), for $k=2,3,4,5$.

Proof: Let $C$ be an arbitrary constant. Then we have

$$
R_{k}(f)=\int_{0}^{1} T_{k}(x)\left[f^{(k)}(x)-C\right] d x=\int_{0}^{1} T_{k}(x) f^{(k)}(x) d x,
$$

for $k=2,3,4,5$, since (13) holds. If we now choose $C=\left(\Gamma_{k}+\gamma_{k}\right) / 2$, then we get

$$
\begin{aligned}
\left|R_{k}(f)\right| & =\left|\int_{0}^{1} T_{k}(x)\left[f^{(k)}(x)-\frac{\Gamma_{k}+\gamma_{k}}{2}\right] d x\right| \\
& \leq \sup _{x \in[0,1]}\left|f^{(k)}(x)-\frac{\Gamma_{k}+\gamma_{k}}{2}\right| \int_{0}^{1}\left|T_{k}(x)\right| d x \\
& \leq \frac{\Gamma_{k}-\gamma_{k}}{2} \int_{0}^{1}\left|T_{k}(x)\right| d x \\
& =\frac{\Gamma_{k}-\gamma_{k}}{2} C_{k},
\end{aligned}
$$


for $k=2,3,4,5$. If we choose $C=\gamma_{k}$, then we have

$$
\begin{aligned}
\left|R_{k}(f)\right| & =\left|\int_{0}^{1} T_{k}(x)\left[f^{(k)}(x)-\gamma_{k}\right] d x\right| \\
& \leq \max _{x \in[0,1]}\left|T_{k}(x)\right| \int_{0}^{1}\left|f^{(k)}(x)-\gamma_{k}\right| d x \\
& =B_{k} \int_{0}^{1}\left[f^{(k)}(x)-\gamma_{k}\right] d x \\
& =\left(S_{k-1}-\gamma_{k}\right) B_{k}
\end{aligned}
$$

for $k=2,3,4,5$.

In a similar way we can prove that (21) holds.

The estimations (19) are Peano-like bounds and they are generally (but not always) better than the usual Peano bounds. Namely, we know that $T_{k}(x)$, $k=2, \ldots, 6$ are Peano kernels. The usual Peano error bounds are

$$
\left|R_{k}(f)\right| \leq\left\|f^{(k)}\right\|_{\infty} \int_{0}^{1}\left|T_{k}(x)\right| d x=C_{k}\left\|f^{(k)}\right\|_{\infty}, \quad k=2, \ldots, 6,
$$

where $\left\|f^{(k)}\right\|_{\infty}=\sup _{x \in[0,1]}\left|f^{(k)}(x)\right|$. If we choose $\gamma_{k}=\inf _{x \in[0,1]} f^{(k)}(x)$ and $\Gamma_{k}=\sup _{x \in[0,1]} f^{(k)}(x)$, then $\frac{\Gamma_{k}-\gamma_{k}}{2} \leq\left\|f^{(k)}\right\|_{\infty}$. Thus, in this case, the error bounds given by (19) are better than the Peano error bounds. In fact, they are equal if and only if $\Gamma_{k}=-\gamma_{k}$. This case $\left(\Gamma_{k}=-\gamma_{k}\right)$ is very rare in practice.

Theoretically, we can derive better error bounds. Let us say something about the last assertion. We can verify that

$$
\int_{0}^{1} T_{k}(x) p_{j}(x) d x=0, \quad j=5-k, \quad k=2,3,4,
$$

where $p_{j}(x)$ is any polynomial of degree $\leq j$. Thus,

$$
\int_{0}^{1} T_{k}(x)\left[f^{(k)}(x)-p_{j}(x)\right] d x=\int_{0}^{1} T_{k}(x) f^{(k)}(x) d x
$$


such that

$$
\left|R_{k}(f)\right| \leq\left\|f^{(k)}-p_{j}\right\|_{\infty} C_{k}, \quad k=2,3,4, \quad j=5-k .
$$

The above estimations are theoretically better than the corresponding estimations in (19).

We now give the above obtained results for an arbitrary interval $[a, b]$. The mapping $x=(t-a) /(b-a)$ is a bijection from $[a, b]$ onto $[0,1]$. If we use this bijection then we find that the polynomials $P_{k}$ and $Q_{k}$ on the interval $[a, b]$ have the forms: $\tilde{P}_{0}(t)=1, \tilde{Q}_{0}(t)=1, \tilde{P}_{1}(t)=t-(23 a+7 b) / 30$, $\tilde{Q}_{1}(t)=t-(7 a+23 b) / 30$, etc. The polynomials can be also obtained by simple integration. For example, $\tilde{P}_{2}$ can be obtained by integrating $\tilde{P}_{1}$ and determining an additional constant such that (13) holds. (They are additionally normalized.) We define the functions

$$
\tilde{T}_{k}(t)= \begin{cases}(-1)^{k} \tilde{P}_{k}(t), \quad t \in\left[a, \frac{a+b}{2}\right) & \\ (-1)^{k} \tilde{Q}_{k}(t), & t \in\left[\frac{a+b}{2}, b\right]\end{cases}
$$

for $k=2, \ldots, 6$. Here we choose $a=x_{i-1}, b=x_{i+1}$ and use the notations introduced in Section 2. Using these functions we derive the following results.

Corollary 4 Let $f \in C^{k}\left(x_{j-1}, x_{j+1}\right), k=2,3, \ldots, 6$. Then we have

$$
\int_{x_{j-1}}^{x_{j+1}} f(x) d x=\frac{7 f_{j-1}+16 f_{j}+7 f_{j+1}}{15} h-\frac{f_{j+1}^{\prime}-f_{j-1}^{\prime}}{15} h^{2}+\tilde{R}_{k}(f),
$$

where

$$
\tilde{R}_{k}(f)=\int_{x_{j-1}}^{x_{j+1}} \tilde{T}_{k}(x) f^{(k)}(x) d x,
$$

for $k=2, \ldots, 6$ and $\tilde{T}_{k}(x)$ defined by (24). 
Corollary 5 Let $f \in C^{k}\left(x_{j-1}, x_{j+1}\right)$ and let $\gamma_{k}, \Gamma_{k}$ be real numbers such that $\gamma_{k} \leq f^{(k)}(x) \leq \Gamma_{k}, x \in\left[x_{j-1}, x_{j+1}\right], k=2,3,4,5$. Let

$$
S_{k}=\frac{f^{(k)}\left(x_{j+1}\right)-f^{(k)}\left(x_{j-1}\right)}{x_{j+1}-x_{j-1}}, \quad k=1,2,3,4 .
$$

Then we have

$$
\begin{aligned}
& \left|\tilde{R}_{k}(f)\right| \leq \frac{\Gamma_{k}-\gamma_{k}}{2} D_{k} h^{k+1}, \\
& \left|\tilde{R}_{k}(f)\right| \leq\left(S_{k-1}-\gamma_{k}\right) E_{k} h^{k+1}, \\
& \left|\tilde{R}_{k}(f)\right| \leq\left(\Gamma_{k}-S_{k-1}\right) E_{k} h^{k+1},
\end{aligned}
$$

where $\tilde{R}_{k}(f)$ are defined by (24), $D_{k}=2^{k+1} C_{k}\left(C_{k}\right.$ are defined by (15)) and $E_{k}=2^{k+1} B_{k}\left(B_{k}\right.$ are defined by (18)), for $k=2,3,4,5$.

Corollary 6 Let $f \in C^{6}\left(x_{j-1}, x_{j+1}\right)$. Then we have

$$
\left|\tilde{R}_{6}(f)\right| \leq D_{6}\left\|f^{(6)}\right\|_{\infty} h^{7},
$$

where $D_{6}=2^{7} C_{6}\left(C_{6}\right.$ is defined by (16)) and $\|g\|_{\infty}=\sup _{x \in\left[x_{j-1}, x_{j+1}\right]}|g(x)|$.

\section{Applications in numerical integration}

We define the partition $\pi=\left\{a=x_{0}<x_{1}<\cdots<x_{2 n}=b\right\}$ of the interval $[a, b]$ such that $x_{i+1}=x_{i}+h, i=0,1, \ldots, 2 n-1, h=(b-a) /(2 n)$. We also define the functions

$$
\bar{T}_{k i}(x)= \begin{cases}(-1)^{k} \bar{P}_{k i}(x), & x \in\left[x_{i-1}, x_{i}\right] \\ (-1)^{k} \bar{Q}_{k i}(x), & x \in\left(x_{i}, x_{i+1}\right]\end{cases}
$$

for $k=2, \ldots, 6, i=1,3, \ldots, 2 n-1$, which correspond to the functions defined by (24) (for $a=x_{i-1}, b=x_{i+1}$ ). 
Theorem 7 Under the assumptions of Corollary 4 suppose that $\pi$ and $\bar{T}_{k}$ are given as above. Then we have

$$
\begin{aligned}
\int_{a}^{b} f(x) d x= & \frac{h}{15} \sum_{j=1, j \text { odd }}^{2 n-1}\left[7 f_{j-1}+16 f_{j}+7 f_{j+1}\right] \\
& -\frac{h^{2}}{15}\left[f^{\prime}(b)-f^{\prime}(a)\right]+\bar{R}_{k}(f)
\end{aligned}
$$

where

$$
\bar{R}_{k}(f)=\sum_{j=1, j \text { odd }}^{2 n-1} \int_{x_{j-1}}^{x_{j+1}} \bar{T}_{k i}(x) f^{(k)}(x) d x,
$$

for $k=2, \ldots, 6$.

Proof: Sum (25) over odd $j$ from 1 to $2 n-1$ to get (31-32), since

$$
\sum_{j=1, j \text { odd }}^{2 n-1}\left[f_{j+1}^{\prime}-f_{j-1}^{\prime}\right]=f^{\prime}(b)-f^{\prime}(a) .
$$

Theorem 8 Under the assumptions of Theorem 7 and Corollary 5 we have

$$
\begin{aligned}
\left|\bar{R}_{k}(f)\right| & \leq \frac{\Gamma_{k}-\gamma_{k}}{4} D_{k} h^{k}(b-a), \\
\left|\bar{R}_{k}(f)\right| & \leq \frac{1}{2}\left(S_{k-1}-\gamma_{k}\right) E_{k} h^{k}(b-a), \\
\left|\bar{R}_{k}(f)\right| & \leq \frac{1}{2}\left(\Gamma_{k}-S_{k-1}\right) E_{k} h^{k}(b-a),
\end{aligned}
$$

for $k=2, \ldots, 5$. 
Proof: The proof follows immediately from Theorem 7 and Corollary 5.

Theorem 9 Under the assumptions of Theorem 7 and Corollary 6 we have

$$
\left|\bar{R}_{6}(f)\right| \leq \frac{1}{2} D_{6} h^{6}(b-a)\left\|f^{(6)}\right\|_{\infty} .
$$

Proof: The proof follows immediately from Theorem 7 and Corollary 6 .

Finally, let us compare the rule obtained in Theorem 7 with the standard composite Simpson's rule

$$
\int_{a}^{b} f(x) d x=\frac{h}{3} \sum_{j=1, j \text { odd }}^{2 n-1}\left[f_{j-1}+4 f_{j}+f_{j+1}\right]+R_{S}(f) .
$$

The terms

$$
\frac{h}{3} \sum_{j=1, j \text { odd }}^{2 n-1}\left[f_{j-1}+4 f_{j}+f_{j+1}\right]
$$

and

$$
\frac{h}{15} \sum_{j=1, j \text { odd }}^{2 n-1}\left[7 f_{j-1}+16 f_{j}+7 f_{j+1}\right],
$$

require a same amount of calculations. The rule (31) has only one additional term with respect to the rule (33), namely

$$
-\frac{h^{2}}{15}\left[f^{\prime}(b)-f^{\prime}(a)\right] .
$$

Hence, the amount of calculations is approximately the same for both formulae. (Recall that function evaluations are generally considered the computationally most expensive part of quadrature algorithms.) On the other hand, the rule (31) is exact for polynomials of degree $\leq 5$, while the rule (33) is 
exact for polynomials of degree $\leq 3$. Furthermore, from Theorem 8 we have

$$
\left|\bar{R}_{4}(f)\right| \leq 2 \frac{\Gamma_{4}-\gamma_{4}}{3645} h^{4}(b-a),
$$

while the standard estimation for the Simpson's rule is

$$
\left|R_{S}(f)\right| \leq \frac{\left\|f^{(4)}\right\|_{\infty}}{180} h^{4}(b-a) .
$$

Since, (34) is better than (35), the rule (31) has better approximation properties than Simpson's rule. Thus, we expect that it will give better results in practice (in most cases).

Example: here we show errors in estimating

$$
I=\frac{\sqrt{\pi}}{2} \operatorname{erf}(1)=\int_{0}^{1} \exp \left(-x^{2}\right) d x .
$$

Using just two subintervals, $h=1 / 2$, our formula (8) computes $I \approx 0.746795$, whereas with four subintervals, that is $h=1 / 4,(8)$ gives $I \approx 0.746824$ which is correct to six decimal places. Figure 1 shows our rule converges quickly with decreasing grid spacing $h$, and is essentially exact to double precision with just 64 subintervals.

\section{References}

[1] P. Cerone and S. S. Dragomir, Midpoint-type Rules from an Inequalities Point of View, Handbook of Analytic-Computational Methods in Applied Mathematics, Editor: G. Anastassiou, CRC Press, New York, (2000), 135-200. E42 


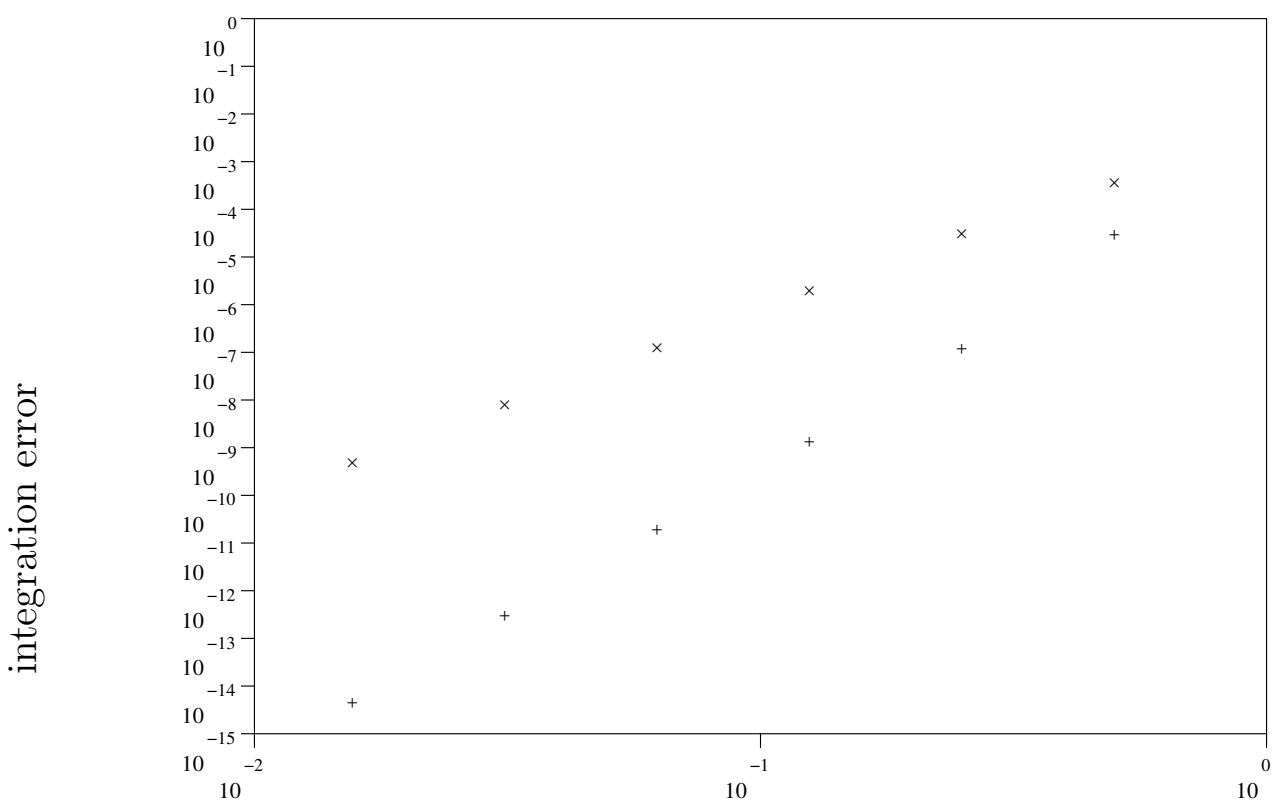

grid spacing $h$

FigurE 1: log-log plot of the errors of our integration rule (8), +'s, as a function showing the $\mathcal{O}\left(h^{6}\right)$ rate of convergence to the erf integral $(36)$, compared to the $\mathcal{O}\left(h^{4}\right)$ convergence of the normal Simpson's rule, $\times$ 's. 
[2] P. Cerone and S. S. Dragomir, Trapezoidal-type Rules from an Inequalities Point of View, Handbook of Analytic-Computational Methods in Applied Mathematics, Editor: G. Anastassiou, CRC Press, New York, (2000), 65-134. E42, E43

[3] P. Cerone, Three Point Rules in Numerical Integration, J. Non-linear Analysis, 47, (2001), 2341-2352. E42

[4] S. S. Dragomir, R. P. Agarwal and P. Cerone, On Simpson's inequality and applications, J. Inequal. Appl., 5 (2000), 533-579. E42

[5] National Physical Laboratory, Modern Computing Methods, volume 16 of Notes on Applied Science, Her Majesty's Stationary Office, 1961. E45 